Musées, Patrimoine et Culture scientifiques et techniques

$144 \mid 2012$

novembre - décembre 2012

\title{
Les citoyens, la biodiversité et le pouvoir
}

\section{Michel Venne}

URL : http://journals.openedition.org/ocim/1147

DOI : 10.4000/ocim. 1147

ISSN : 2108-646X

\section{Éditeur}

OCIM

Édition imprimée

Date de publication : 1 novembre 2012

Pagination : 60-66

ISSN : 0994-1908

Référence électronique

Michel Venne, "Les citoyens, la biodiversité et le pouvoir », La Lettre de l'OCIM [En ligne], 144 | 2012, mis en ligne le 01 novembre 2014, consulté le 01 mai 2019. URL : http://journals.openedition.org/ ocim/1147; DOl : 10.4000/ocim.1147 
On risque d'affaiblir ainsi les mécanismes de détermination du bien commun. Les citoyens n'acceptent plus d'être gouvernés par des oligarchies qui ne tiennent pas compte de leurs priorités. Ils ne croient plus dans des vérités divines. Ils demandent à être convaincus, inclus dans la délibération de laquelle naît les conscensus requis pour vivre ensemble.

Dans ce court article, nous souhaitons réfléchir sur la place des citoyens dans ces démocraties délibératives, dans lesquelles les élus, les acteurs socio-économiques mais aussi les scientifiques continuent certes de jouer un rôle de premier plan, mais doivent désormais composer avec celui de populations plus scolarisées, mieux informées et en même temps bien plus diversifiées et difficiles à représenter qu'autrefois. Nous souhaitons ensuite exposer certains des principes à respecter pour favoriser une participation citoyenne qui soit crédible, légitime, utile et efficace, et ce à partir d'un des projets menés par l'INM en collaboration avec Espace pour la vie, à Montréal.

\section{Un principe du développement durable}

La participation du public est un principe inhérent au développement durable. « La participation et l'engagement des citoyens et des groupes qui les représentent sont nécessaires pour définir une vision concertée du développement et assurer sa durabilité sur les plans environnemental, social et économique », affirme la Loi québécoise sur le développement durable ${ }^{(2)}$.

L'apport du public prend plusieurs formes. Les citoyens agissent directement sur leur milieu de vie. Leur action peut contribuer à le détériorer ou à le protéger. Si l'État peut créer les conditions pour soutenir et encourager des comportements responsables, seuls les citoyens sont garants du succès de plusieurs de ces politiques, de la collecte sélective des déchets à l'achat local de produits équitables et bio, jusqu'à l'efficacité énergétique de leur propre maison ou l'embellissement de leur quartier.

Les citoyens sont aussi les meilleurs gardiens de la qualité des milieux de vie qu'ils ont en partage. Ils sont les vigiles les plus efficaces de la qualité de l'eau potable, de la préservation des écosystèmes des bassins versants, de la protection des espèces vulnérables dans les milieux humides menacés par les développements immobiliers. Nul autre que l'agriculteur connaît mieux que lui les soins qu'il faut prodiguer au sol pour que celui-ci demeure fertile. Le chasseur ou le trappeur amérindien sait l'importance de la biodiversité en forêt. Le pêcheur vit de la préservation des écosystèmes marins. L'ornithologue ou le botaniste amateur est le meilleur ami de la nature.

Ce sont des citoyens, réunis en associations, en comités ou en mouvements qui, en mettant en commun leurs connaissances et leurs volontés, alertent l'opinion publique sur les périls qui menacent l'environnement ou la justice sociale. Ils aiguillonnent les décideurs publics. Ils surveillent les forces du marché, les grandes et petites entreprises, dénoncent les pratiques douteuses. Ils ont, à cet égard, le pouvoir de salir une réputation, de faire baisser la valeur des actions en bourse ou de faire fuir la clientèle des compagnies qui se comportent en mauvais citoyens corporatifs. Ils ont aussi celui de forger des normes sociales.

Enfin, qui élit les gouvernements ? Qui choisit ceux qui adoptent les lois, édictent les règles du jeu ? Ce sont les citoyens, bien sûr. Et leur choix n'est pas anodin. Le Canada, autrefois champion de la lutte contre les changements climatiques est désormais au ban avec les États voyous. Ce sont ces citoyens aussi qui, entre les élections, se présentent aux consultations parlementaires ou dans les processus d'évaluation environnementale. Comme ceux que j'ai vus, dans des consultations que j'ai eu le bonheur de présider, mettant leurs compétences au service de la collectivité, présenter des dossiers étoffés, fondés sur de la connaissance scientifique, pour remettre en question la filière gazière, dénoncer un projet domiciliaire à l'orée d'un parc national ou refuser de laisser aux compagnies minières l'accès illimité à leur territoire.

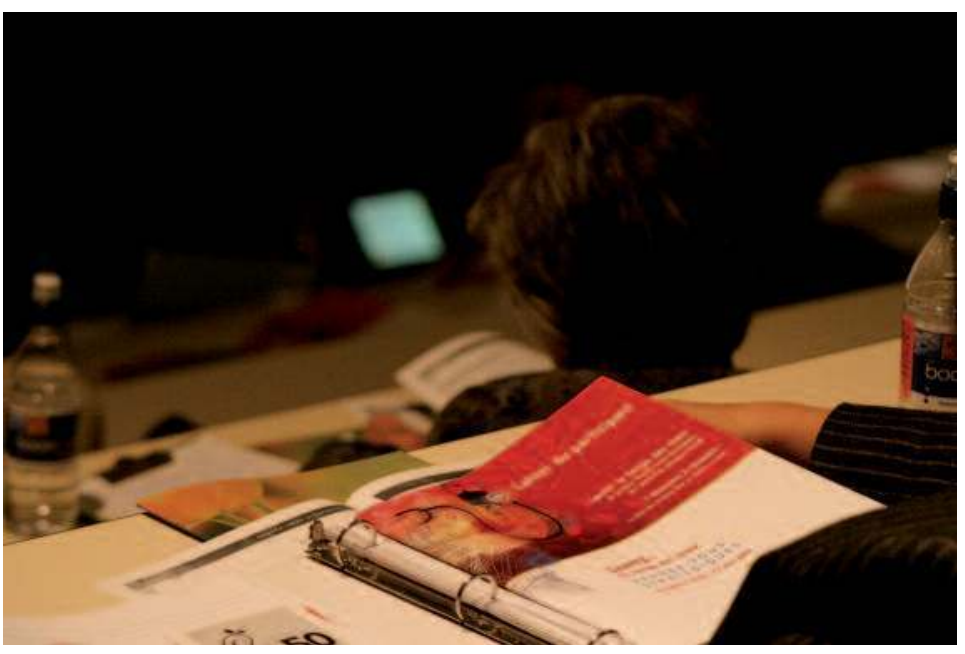

Dans ces débats sociétaux, un cahier remis aux participants permet d'alimenter la discussion générale. 


\section{Le dialogue essentiel entre la science et la société}

La participation citoyenne se réalise à l'intérieur de rapports de force, évidemment. Participer n'est pas l'apanage des gens de gauche, pas plus que de droite. Les citoyens font des choix qui sont certes fondés sur des informations mais aussi sur des croyances ou des valeurs. La participation citoyenne conteste l'influence déterminante des bureaucrates sur les décisions administratives et politiques. Elle conteste aussi le monopole des scientifiques sur la connaissance et la détermination de la vérité.

En soi, cette idée que le monopole scientifique se lézarde sous les coups de boutoir des manifestations citoyennes, serait une raison suffisante pour que les scientifiques comprennent l'impérieuse nécessité d'entrer en dialogue avec ceux, les citoyens, qui constituent une force difficile à dompter mais dont la puissance peut appuyer le progrès de l'humanité ou, au contraire, contribuer à sa perte.

Plus personne, dans le monde scientifique, ne peut prétendre aujourd'hui pouvoir imposer unilatéralement une vérité. Les OGM sont-ils ou non une bonne chose? Doit-on exploiter les gaz de schiste? Les ondes émises par nos téléphones cellulaires sont-elles nocives pour la santé ? Le tabagisme est-il aussi dangereux qu'on le dit ? Le réchauffement de la planète est-il un enjeu véritable ? À aucune de ces questions la science n'est en mesure de trouver une réponse. La conclusion qui s'imposera, le sera par divers processus politiques. Ces processus sont traversés par des combats entre des groupes d'intérêt.

Le minimum que les scientifiques peuvent faire, c'est de veiller à ce que tous ces groupes, de veiller à ce que la population en général, aient accès à la connaissance la plus exacte, la plus à jour, la plus utile possible pour résoudre les dilemmes de plus en plus complexes auxquels l'humanité est confrontée.

Il nous semble également du devoir des scientifiques de mettre en échec des interprétations s'appuyant soi-disant sur la science mais qui sont de pures fabulations. Or les scientifiques n'ont pas tous appris, encore, à dialoguer avec le peuple et avec les puissances de la société, notamment celles de l'argent. Nous parlons ici de quelque chose qui va bien au-delà de la vulgarisation et du documentaire animalier. Ce dialogue entre la science et la société est devenu une condition préalable de démocratie.

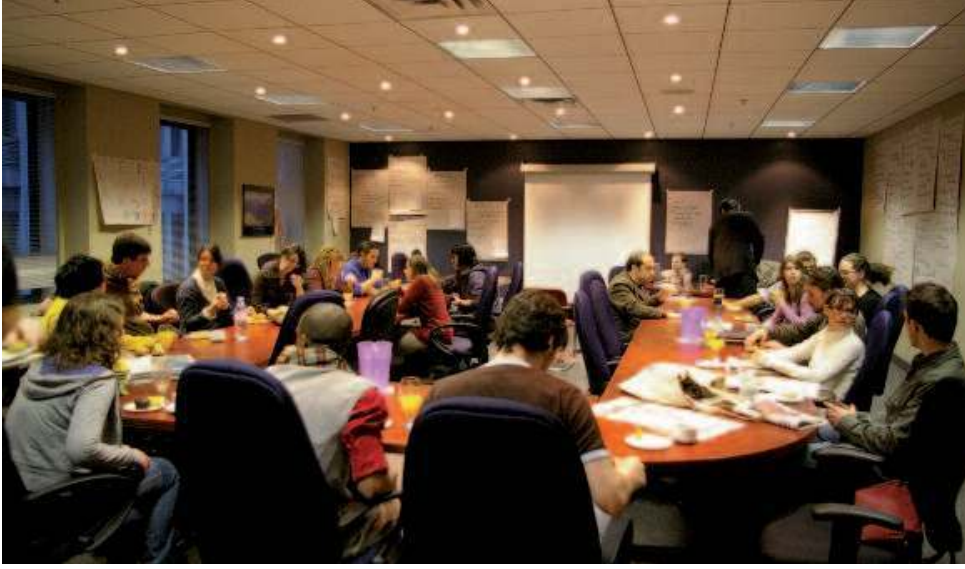

La délibération permet de clarifier les valeurs en cause, au participant d'écouter le point de vue des autres et ainsi de se remettre en question : séance de travail des jeunes de I'École d'été de l'Institut du Nouveau Monde en 2008 pour la rédaction de la Déclaration jeunesse de Québec. () INM

\section{La science est entrée en politique}

La science est entrée en société depuis longtemps. La découverte du moteur à vapeur, de l'imprimerie, du transistor ou du microprocesseur, ont changé le visage de l'humanité. La majorité des progrès économiques récents découlent de découvertes scientifiques ou de leur application à grande échelle. Nous sommes entrés, dit-on, dans une société du savoir. L'invention de la bombe atomique et son usage à des fins militaires par les Américains, contre le Japon, à la fin de la deuxième guerre mondiale, marquent toutefois, dans les imaginaires, l'entrée de la science en politique. Des milliers de scientifiques ont alors été mobilisés pour doter les États-Unis d'un moyen militaire inédit. La science perdait son innoncence. Celle-ci n'était plus seulement porteuse de progrès mais aussi de destruction. Depuis, la science ne peut pas échapper à l'examen public et à un débat démocratique concernant ses fins et les moyens qui lui sont attribués.

Depuis, une certaine méfiance s'est emparée de la population à l'égard de ce que l'on appelle la technoscience. Nous sommes entrés depuis quelques décennies dans ce que des sociologues appellent des « sociétés $d u$ risque» (3). Nous devons apprendre comme sociétés à évaluer les risques que nous partageons et à composer avec eux. Il faut inventer de nouveaux lieux d'arbitrage et d'évaluation du risque partagé. C'est la pratique démocratique elle-même qui doit être réinventée et ce, en prenant garde à éviter le triple piège de conforter le pouvoir de groupes d'intérêt au détriment du bien commun, de reproduire dans des instances délibératives les inégalités sociales existant dans la société ou d'instituer des républiques de bavardage qui ne servent à rien d'utile. Enfin, dans le contexte d'une société du savoir, il importe de veiller au développement d'une culture 
scientifique et d'une relève scientifique. Le système d'éducation est évidemment interpellé mais d'autres acteurs le sont également, dont les musées.

\section{La gouvernance collaborative}

Il est démontré par l'expérience internationale que la participation civique contribue à améliorer les décisions, les programmes et les services par la prise en compte d'éclairages diversifiés ; assure une meilleure compréhension des enjeux et une meilleure identification des problèmes ; force la transparence des acteurs intéressés et favorise ainsi une meilleure reddition des comptes ; aide à identifier les aspects controversés et les consensus viables ; favorise le soutien populaire aux politiques publiques ; développe les capacités du public (empowerment); et favorise la cohésion sociale et le sens civique ${ }^{(4)}$. Toute la question est de savoir comment structurer cette participation civique.

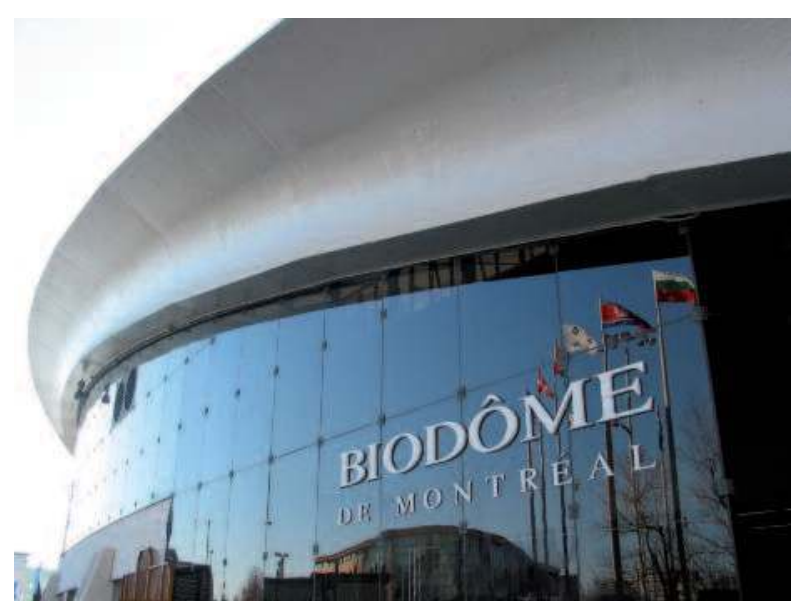

Entrée du Biodôme (c) Biodôme

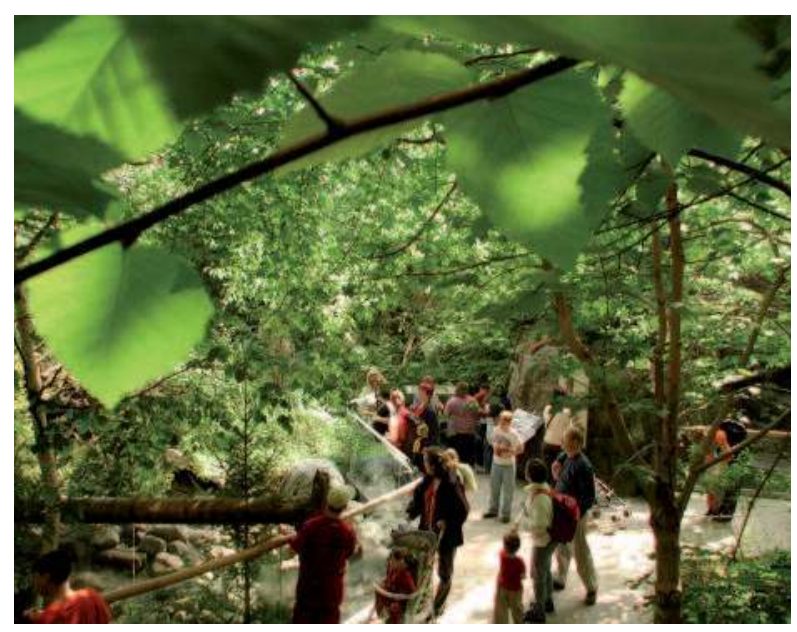

Le sentier de la forêt laurentienne au Biodôme (c) Biodôme
Le vocable employé désormais pour désigner le consensus autour d'un projet est celui d'acceptablilité sociale. Or celle-ci s'obtient dans le cadre de processus de participation citoyenne et de concertation des parties prenantes ${ }^{(5)}$. L'élaboration des politiques publiques en vase clos, respectant un processus décisionnel linéaire, ne répond plus aux nouvelles réalités (6).

Le concept de gouvernance collaborative traduit bien la direction que nos sociétés doivent prendre. Dans une perspective d'administration publique, Ansell et Gash ${ }^{(7)}$ définissent la gouvernance collaborative comme un arrangement dans lequel une ou des organisations publiques engagent une collaboration avec des parties prenantes non étatiques dans un processus de décision collectif formel, orienté vers le consensus, délibératif et qui a pour but de définir ou de mettre en œuvre des politiques publiques ou de gérer des programmes ou des actifs publics.

Plus globalement, la gouvernance renvoie à « un processus de coordination d'acteurs, de groupes socianx et d'institutions, en vue d'atteindre des objectifs définis et discutés collectivement. La gouvernance renvoie alors à l'ensemble d'institutions, de réseanx, de directives, de réglementations, de normes, d'usages politiques et sociaux, ainsi que d'acteurs publics et privés qui contribuent à la stabilité d'une société et d'un régime politique, à son orientation, à la capacité de diriger, et à celle de fournir des services et à assurer sa légitimité » (8).

Cette idée de la gouvernance collaborative s'applique autant dans les cas d'adoption de grands projets de développement quà l'échelle locale. Depuis 2003, l'INM a expérimenté diverses approches participatives et délibératives incluant une participation des citoyens sur des enjeux aussi délicats que l'intégration des immigrants ou le développement de la filière gazière dans la vallée du Saint-Laurent, dans le cadre de processus locaux de planification ou pour l'adoption d'un Agenda 21 de la culture pour le Québec, pour ne nommer que ceux-là.

Ces derniers mois, l'INM a proposé à Espace pour la vie, le plus important complexe muséal en sciences naturelles au Canada, situé en plein cœur de Montréal, une démarche participative et délibérative pour élaborer une stratégie de participation citoyenne. Cet exemple paraît particulièrement bien choisi pour illustrer les exigences et les modalités d'un processus participatif et délibératif conduisant à l'adoption d'une politique, d'une stratégie ou d'un programme. 


\section{Cocréation : s'informer, débattre, proposer}

La démarche qui a été proposée s’inspire des approches développées par l'Institut du Nouveau Monde depuis sa création. Elle comporte un moment d'information, un moment de délibération qui débouche sur des propositions. S'informer, débattre, proposer sont les trois verbes, déclinés selon mille et une méthodologies participatives et délibératives, qui composent l'approche de base de l'INM. S'informer parce qu'on ne peut débattre de choses qu'on ne connaît pas. Débattre parce qu'une opinion ne vaut rien si elle ne subit pas avec succès le test de la délibération. Proposer parce qu'il ne sert à rien de bavarder sur un sujet par ailleurs fort intéressant si cela ne conduit pas à une conclusion prenant la forme d'une recommandation, d'un projet, bref, d'un résultat ${ }^{(9)}$.

Espace pour la vie se veut un espace de cocréation façonné par les Montréalais et les visiteurs du monde entier. Cette vision s'incarne non seulement dans l'animation des quatre institutions muséales (Biodôme, Insectarium, Jardin botanique, Planétarium) mais plus particulièrement dans l'aménagement (d'ici 2017) de la Grande Place qui reliera les quatre institutions. Cette esplanade offrira une expérience intégrative qui symbolise la relation entre l'être humain et la nature, une agora en perpétuelle évolution. Sur les lieux, les visiteurs pourront participer à la plantation de vivaces, habiter les lieux dans une yourte en buvant un chocolat chaud fait de cacao équitable, jogger, visionner un film, discuter avec des groupes, créer un club de tricot hivernal, y travailler avec son portable à l'ombre des pommetiers en fleurs, bâtir un pavillon éphémère, réfléchir collectivement et expérimenter des technologies vertes...

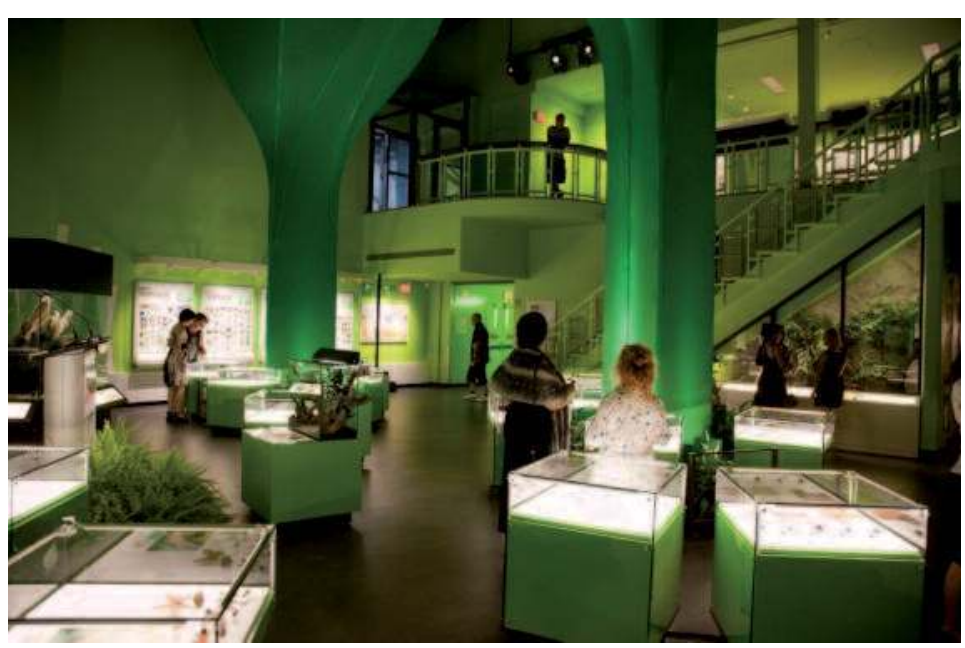

Salle d'exposition de l'Insectarium (๑) Mediumres/Vincenzo D'Alto
L'approche proposée par l'INM pour inclure les citoyens dans le développement de cet espace s'appuie sur quatre éléments-clés :

- la connaissance scientifique et les pratiques exemplaires ;

- un travail collaboratif ;

- la cocréation des diverses parties prenantes;

- l'appropriation du projet par les participants.

La rédaction du cadre stratégique s'appuiera sur une connaissance scientifique autant sur le plan théorique que pratique. Nous proposons d'interpeller, par l'entremise d'un comité scientifique interdisciplinaire composé d'experts, les théories de la participation citoyenne, de la concertation et de l'animation sociale, et des disciplines comme l'architecture, l'aménagement et l'urbanisme, la muséologie, la récréologie...

Nous proposons de recenser non seulement les théories mais aussi les pratiques exemplaires observées dans des situations similaires à celles proposées par Espace pour la vie à travers le monde. Cette recherche pourra être complétée par la mobilisation de communautés d'experts internationaux en utilisant un outil de collaboration en ligne (de type wiki) spécialement conçu à cette fin.

Nous proposons de former un Comité directeur, constitué de membres du personnel désignés par Espace pour la vie, un expert du comité scientifique, cinq représentants des communautés d'appartenance d'Espace pour la vie (voir le paragraphe sur la cocréation) et l'équipe de l'INM pour encadrer les travaux de rédaction du cadre stratégique. Ce groupe de référence aurait pour mandat d'ouvrir les pistes initiales de réflexion et de valider les contenus rassemblés aux fins de la rédaction des documents, ainsi que les méthodologies choisies pour inclure les parties prenantes dans la démarche.

Nous recommandons vivement que le processus de rédaction du cadre stratégique qui est également présenté comme "plan de cocréation d'Espace pour la vie » soit lui-même, dès le départ, conçu comme une démarche de cocréation impliquant les parties prenantes des communautés d'appartenance d'Espace pour la vie.

Nous proposons de constituer cinq groupes de travail composés d'une dizaine de membres chacun, sélectionnés sur appels à candidatures. Chacun des cinq groupes de travail correspond à l'une des principales communautés d'appartenance d'Espace pour la vie, soit :

- les employés des quatre institutions d'Espace pour la vie (sélectionnés à partir d'un appel à candidatures conçu en collaboration avec les syndicats d'employés); 


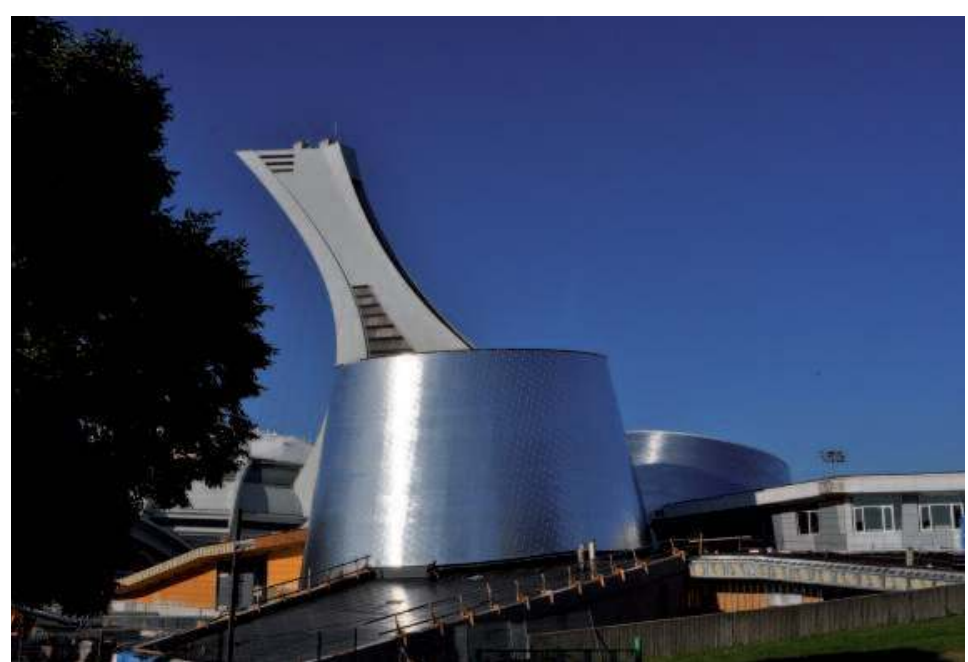

Le Planétarium (au premier plan) (c) Johanne Landry

Pour réussir une démarche participative, il faut se rendre là où les citoyens vivent et se sentent à l'aise ; rendre l'information disponible sur des supports diversifiés en fonction des publics ; consacrer beaucoup d'énergie à la phase de recrutement des participants pour en assurer la diversité ; prévoir plusieurs modalités de participation adaptées aux différents publics ; proposer des procédures conviviales (avoir du plaisir à participer).

La participation citoyenne est particulièrement utile et efficace pour la formulation des politiques publiques : - lorsque les normes sociales sont devenues inadéquates ou inexistantes, la participation permet de faire émerger de nouvelles normes par le dialogue ;

- lorsque le débat est monopolisé par les groupes d’intérêt, la participation permet alors de faire apparaître la pluralité des points de vue ;

- lorsqu'une question requiert un arbitrage entre des valeurs ou la clarification de principes, la sensibilisation et l'apprentissage du public à propos de questions politiques nouvelles ou complexes et dont la solution, pour être efficace, requiert une adhésion morale forte ; - lorsqu'une décision relève de la gestion d'un risque partagé ou concerne l'implantation d'une innovation.

Enfin, on peut dire qu'il y a des démarches de participation qui sont plus porteuses que d'autres, ce sont celles qui incluent une véritable délibération et celles qui s’inscrivent dans la durée. La délibération permet de clarifier les valeurs en cause, au participant d'écouter le point de vue des autres et ainsi de se remettre en question voire de changer d'avis. Dans la délibération, les intérêts communs dominent généralement les intérêts particuliers. Lorsqu'une démarche s'inscrit dans la durée, elle permet au participant de s'approprier le sujet, d'acquérir des compétences, et d'instaurer un dialogue, une mémoire, une sagesse.

\section{Notes}

(1) Venne, M. Des citoyens responsables, Télescope, vol. 17, n¹, 2011, pp.194-212. http://telescope.enap.ca/Telescope/docs/Index/Vol_17_nol/ Telv17nl_venne.pdf

(2) Principe de la loi sur le Développement durable, L.R.Q., chapitre II, article 6 .

(3) Beck, U. La Société du risque. Sur la voie d'une autre modernité. Paris : Alto, 2001, $516 \mathrm{p}$

(4) Mercier, C., Bourque, D. et Saint-Germain, L. Participation citoyenne et développement des communautés au Québec : enjeux, défis et conditions d'actualisation. Alliance de recherche université-communauté (ARUC) Innovation sociale et développement des communautés et universitié du Québec en Outaouais, 2009

(5) Côté, L. Éditorial, Télescope, vol. 17, n¹, 2011, pp. 3-5.

(6) Blondiaux, L. et Fourniau, J.-M. Démocratie et participation : un état des savoirs, Bruxelles, Participations, $n^{\circ} 1,201$ 1, pp. 1-347.

(7) Ansell, C. et Gash, A. Collaborative Governance in Theory and Practice, Journal of Public Administration Research and Theory, $\mathrm{n}^{\circ} 18$ (4), 2008, pp. 543-571

(8) Le Galès, P. Gouvernance, in Dictionnaire des politiques publiques, Boussaguet, L., Jacquot, S. et Ravinet, P. (dir.). Paris : Presses de Sciences Po, 2004, pp. 242-250.

(9) L'INM s’inspire du Spectrum de la participation du public élaboré par l'association internationale de la participation du public (AIP2) en 2007. www.iap2.org/associations/4748/files/IAP2\%20Spectrum_vertical.pdf (10) Fondation Roi Beaudoin Méthodes participatives, un guide pour l'utilisateur. Bruxelles, 2006, 181 p. 